http://dx.doi.org/10.21611/qirt.2006.016

\title{
A neural approach for thermographic image analysis
}

\author{
T. D'Orazio ${ }^{a}$, M. Leo ${ }^{a}$, A. Distante ${ }^{a}$, \\ V. Pianese ${ }^{b}, G$. Borzacchiello ${ }^{b}, G$. Cavaccini ${ }^{b}$ \\ ${ }^{a}$ CNR-ISSIA via Amendola 122/D-I, 70126 BARI ITALY \\ bAlenia Aeronautica v.le dell'Aeronautica, 80038 Pomigliano D'Arco (Napoli) ITALY
}

\begin{abstract}
The analysis of the internal defects (not detectable by a visual inspection) of the aircraft composite materials is a difficult task unless invasive techniques are applied. In this paper we have addressed the problem of inspecting composite materials by using automatic analysis of thermographic techniques.

The proposed approach consists of two steps: at first a neural network was trained to model the time space variations in a sequence of thermgraphic images and then the same neural network was applied to all the points of a sequence of thermographic images. The experimental tests were performed on a composite material and they demonstrate the ability of the method to recognize regions containing defects even in presence of considerable lighting variations.
\end{abstract}

Keywords: Infrared thermography, internal defects, neural networks, defect classification

\section{Introduction}

The problem of guarantying reliable and efficient safety checks has received great attention in recent years in aeronautic contexts: the maintenance operations, especially those applied on in-service aircraft, have to be reliable but also have to be performed at low cost in order to meet frequent schedules. In particular non destructive testing and evaluation (NDT\&E) techniques are necessary to early detect damage in high stressed and fatigue-loaded regions of the structure. Some of these NDT\&E techniques are based on analysis of the transmission of different signals such as ultrasonics, acoustic emission, thermography, laser ultrasonic, Xradiography, eddy currents, shearography, and low frequency methods [1]. 
Transient thermography is a very promising technique for the analysis of aircraft composite materials [2]. It is a non-contact technique which uses the thermal gradient variation to inspect the internal properties of the investigated area: the materials are heated by some external source (lamps) and the resulting thermal transient is recorded using an infrared camera. Some research has been presented in the literature on the use of thermography to investigate aircraft components $[3,4,5]$. They have demonstrated the effectiveness of thermography in detecting internal defects and show excellent results on all the investigated samples.

Different qualitative approaches have been developed by many researchers to investigate the effects on thermographic images of a number of parameters such as: specimens of materials, defect types, depths of defect, size and thickness $[6,7,8]$.

Quantitative approaches are attractive in the analysis of thermographic images because of the possible diagnosis capabilities that they introduce. They involve the solution of the direct problem, that is the computation of the expected response from known sound and defect materials, and the inverse problem, that is the evaluation of defect characteristics from a known response. Due to the nonlinear and non-univocal nature of these mapping problems, the solution is rather complex. For this reason some attempts using neural networks have started to emerge in the last few years $[9,10,11]$.

In this paper we address the problem of developing an automatic system for the analysis of sequences of thermographic images to help the safety inspector when elaborating his diagnosis. Starting from the observation that composite materials have different behaviors during the transient phase of the thermographic inspection, we devised a neural approach that learns the main characteristics of these thermic evolutions and then uses them to classify the investigated area as a defective or sound area. The neural network classifiers were found to be particularly effective since they can easily implement the non linear mapping from an input feature space to an output space.

In this work a composite sandwich material with a metallic core was considered. When the material is irradiated with high power lamps its surface has variable thermic gradients that can be recorded with a thermo-camera. The reflectivity time evolution of each pixel of the image differs when some regions contain inner defects due, for example, to water intrusion or holes caused by impact. A supervised learning method was used to train the neural network to analyze the variations of these mono dimensional signals and to extract the main characteristics associated to different 


\section{http://dx.doi.org/10.21611/qirt.2006.016}

regions of the same materials. To reduce the effects of noise and lighting variation, denoising and normalizing algorithms were applied to the initial signals. The neural network was trained to recognize different defects by using a sample set of image points. The whole system was tested on all the image pixels simulating different linear and non linear lighting variations.

Experimental results on the considered composite materials demonstrate the effectiveness and the potential capabilities of the proposed approach even in presence of considerable lighting variations.

The structure of this paper is as follows. In Section 2, the system overview and the experimental setup are presented. The neural network for classification of defects is described in Section 3 whereas, in Section 4, the experimental results are presented.

\section{System overview}

Thermographic analysis is based on estimating the reflectivity of the materials undergoing temperature variations. Usually, the considered spectrum is centered at 5-6 $\mu \mathrm{m}$ (short wave band) or centered at $10 \mu \mathrm{m}$ (long wave band). The detection is carried out by using thermo-cameras sensitive to the infrared emissions. Of course, this kind of analysis is only applicable to materials that have a good thermic conductivity such as metals and carbonic composites. Different types of thermic excitation can be used according to the materials and the defects considered: for instance uniform heating, spot heating, line heating. In this work, a quasi-uniform heating has been guaranteed by using Xenon quartz lamps with a power between $250-375 \mathrm{~W}$. This kind of heating allows a temperature variation around $20^{\circ} \mathrm{C} / \mathrm{sec}$. In figure 1 the lamps and the thermocamera used for the experimental setup are shown. In this work a sandwich material was analyzed: it has an external skin in carbon fibre composite and a metallic core. The analyzed structure has three holes with different diameters and depths in which different quantities of liquid were inserted. In the boundary areas of these holes the presence of vapor is evident because of the phase transformation from liquid to gas. In figure 2 it is possible to observe the analysed structure and one of the corresponding thermographic images. On the right the gray level scale corresponding to the temperature of the material is reported.

In all the cases the defects or the internal damage are not detectable with a visual inspection. 
In the experiments carried out on this material the maximum power of the lamps and the duration of the heating were $5 \mathrm{~kW}$ and 8 seconds. We have analyzed monodimensional signals obtained by considering the time variation of each pixel in the sequence of thermographic images. For each point (i,j) of the image (see fig. 5) the mono-dimensional signal is generated from the gray levels of the same point in the sequence of images: this signal represents the temperature variation of the material during and after the heating process. For example in figure 6 , two points of the analyzed structure are shown. The first one corresponds to a defective point while the second one corresponds to a sound point. On the $x$ axis are the frame numbers, on the $y$ axis the corresponding gray levels that depend on the temperature of the material.

In this way it is possible to generate spatio-time variant images the analysis of which allows the evaluation of the thermic gradient during the heating process. In figure 6 the two investigated points show different behaviors: the defective point presents a reduction of the temperature in the first 50 frames because of the phase transition from liquid to vapor and then an increase of the temperature in the successive frames. The second point signal, corresponding to a sound area, presents an increase of the temperature in the first 50 frames during heating; when the lamps are switched off the temperature decreases.

However it is clearly evident that a functional description of the intensity variations cannot be easily generalized for the considered material and the behaviors of the points corresponding to defective areas are not similar. For this reason we have considered the use of a neural network trained to characterize and recognize the temporal variations of different materials without any specific knowledge of the resulting curves. It is only necessary to select a set of sample points from known defective and no-defective areas. If new classes of defects are encountered, the approach will be able to distinguish them from points belonging to sound material.

\section{The neural network classifier}

A neural network is a massively parallel distributed processor that has a natural propensity for storing experiential knowledge and making it available for use. The knowledge is acquired by the network through a learning process. Interneuron connection strengths, known as synaptic weights, are used to store knowledge [12]. The large success of neural networks derives from their ability to solve complex 
http://dx.doi.org/10.21611/qirt.2006.016

problems that require a non-linear mapping between the input space and the output space, and their generalization capability that allows them to produce reasonable outputs for inputs not encountered during learning.

In this work we have used a Multi Layer Perceptron characterized by the presence of an input layer of source nodes, a hidden layer and an output layer. The hidden layer enables the network to extract higher-order statistics especially when the size of the input layer is large. Supervised learning involves applying a set of training examples to modify the synaptic weights connecting the neurons of the network. Each example consists of a unique input signal and the corresponding desired response. The network is presented many examples many times and the synaptic weights are tuned so as to minimize the difference between the desired response and the actual response of the network. The network training is repeated until a steady state is reached, where there are no further significant changes in the synaptic weights.

The input layer has a number of neurons equal to the number of image features. In this work the features are those extracted after the pre-processing phase (to be discussed in the next section). The number of nodes in the output layer depends on the number of classes that the network has to recognize. In our context the considered composite material present only one kind of defect, and so the network recognizes the sound point and the defect points ( 2 output nodes). The number of nodes in the hidden layer is experimentally determined (in our context 120 hidden nodes was the best choice).

\section{Experimental results}

The length of the thermographic sequence relative to the considered composite material was 207 images.

In the experimental phase, at first, a set of training examples was fixed for the considered composite material and the network was trained using the well known Back Propagation algorithm [13]. In particular 20 examples belonging to the sound area and 30 examples belonging to the defective area were selected.

After the training phase, all the points of the thermographic sequences were provided as input to the net in order to classify them as belonging either to the sound or to the defective area. 
In order to establish the membership class of each element of the test set, the activation level of each output node at the end of the training procedure was evaluated and a set of proper minimum thresholds was introduced.

In the following test phase, when the output of the net does not satisfy this minimum threshold the correspondent test signal was classified as belonging to an ambiguous class.

In both training and test phases, before neural analysis, the signal of the thermic variation for each pixel was, at first, smoothed by a median filter in order to cut down noise frequencies and then normalized maintaining all the pixel values in the range $(0,1)$. In this way the analysis of the thermic variations became independent from the maximum values of the thermic curves and it can be characterized only on the kind of the temporal variation.

This smoothed and normalized signal of the thermic variations was provided to the input layer of the neural network.

The results for the considered composite material are shown in figure 5.

Different colors have been used to represent the results of the classification. The regions around the defect areas are those classified as ambiguous, that actually correspond to the contour of defects.

In order to evaluate the capability of the proposed approach to generalize knowledge additional experiments where carried out. In particular linear lighting variations where simulated in the test phase (leaving unchanged the training set). Linear lighting variations had the analytic description as

$I^{*}=a \times I$

where $I^{*}$ is the new intensity value, $l$ is the old intensity value and $a$ is the lighting gain varying in the range $(0,2)$. Figure 6 shows the classification results when a $20 \%$ darkening (figure 6.a) or brightening (figure 6.b) was introduced. Results with different lighting parameters were performed $(50 \%, 90 \%)$ but are not reported in the paper for lacking of space.

The classification performance remained satisfactory also in presence of strong lighting variations even if, in presence of brightening, it was not always possible to detect all the defect regions and, in addition, the uncertainty area increased. 


\section{Conclusion and Future Works}

A large amount of research work has been conducted using various NDT \& $E$ techniques in the detection and identification of detects in aircraft parts. The thermographic inspection of composite materials has been proven to be effective in the assessment of internal defects of aircraft composites. However, no much work has been done in the automatic inspection of the observed thermic phenomenon. In this paper we have presented a neural approach for the automatic analysis of thermic image sequences and the detection of internal defects of composite materials. The system requires only the selection of a set of training examples extracted from defect areas and sound area. The neural network is able to build an internal representation of the mapping from the input signals to the desired output, and is able to generalize to new situations never encountered in the training phase. Experimental results carried out on a composite material have demonstrated the effectiveness of the proposed approach even in presence of lighting variations.

Future work will be addressed to experiments with unsupervised classifiers to shorten the initial training phase and recognize the defect areas and the sound area without any example of the desired behavior.

\section{References}

[1] Y.D. Huang, L. Froyen, M. Wevers Quality Control and Nondestructive Test in Metal Matrix Composites. Journal of Nondestructive Evaluation, 2001; 20 (3): 113132

[2] G. Gaussorgues, Infrared Thermography. Champan\& Hall, 1994

[3] T.S. Jones, Infrared Thermographic evaluation of marine composite structures, SPIE Vol. 2459; 1995.

[4] N.P.Avdelidis, B.C. Hawtin, D.P. Almond. Transient thermography in the assessment of defects of aircraft composites. NDT \& E Int. 2003; 36: 433-439.

[5] D.Wu, G. Busse. Lock-in thermography for nondestructive evaluation of materials. Rev. Gen. Therm. 1998; 37, 693-703.

[6] T. Sakagami, S. Kubo. Applications of pulse heating thermography and lock-in thermography to quantitative nondestructive evaluations. Infrared Physics \& Technology. 2002; 43:211-218.

[7] G.Giorleo, C. Meola, A. Squillace, Analysis of Detective Carbon-Epoxy by Means of Lock-in Thermography. Res. NonDestr. Eval 2000, 241-250 
[8]T. Inagaki, T. Ishii, T. Iwamoto On the NDT and E for the diagnosis of defects using infrared thermography. NDT \& E Int. 1999; 32:247-257.

[9] X. Maldague, Yves Largouet, J.P. Couturier. A study of defect using neural networks in pulsed phase thermography: modeling, noise, experiments. 1998; 37:704-717.

[10] J.Y. Marin, H. Tretout. Advanced technology and processing tools for corrosion detection by infrared thermography. AITA- advanced Infrared Technology and Appliation 1999, 128-133.

[11] M.B.Saintey, D.P:Almond. An artificial neural network interpreter for transient thermography image data. NDT \& E Int. 1997; 30(5):291-295.

[12] S. Haykin , Neural Network a comprehensive foundation, IEEE Press 1994

[13] J. Freeman, D. Skapura, Neural Network Algorithms, Applications, And Programming Techniques, Addison Welsey 1991

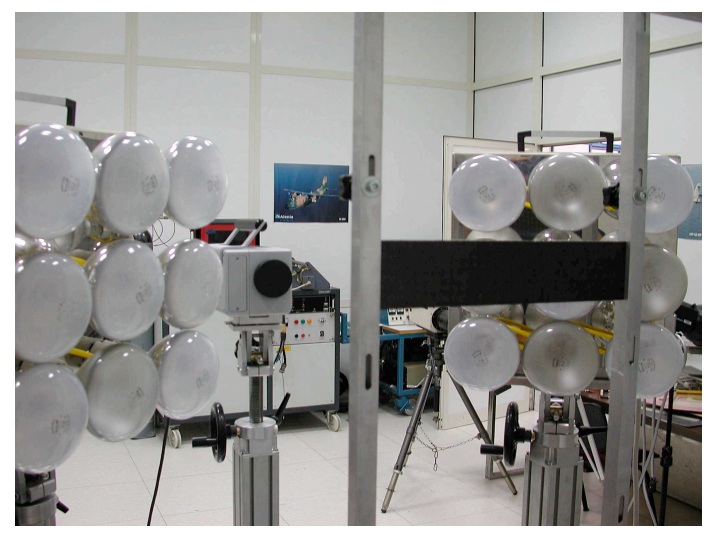

Figure 1- Lamps and thermocamera used for the experimental setup

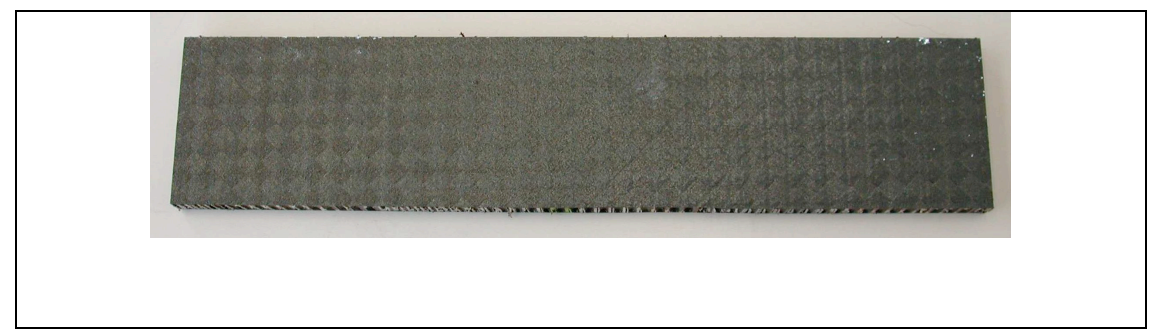


http://dx.doi.org/10.21611/qirt.2006.016

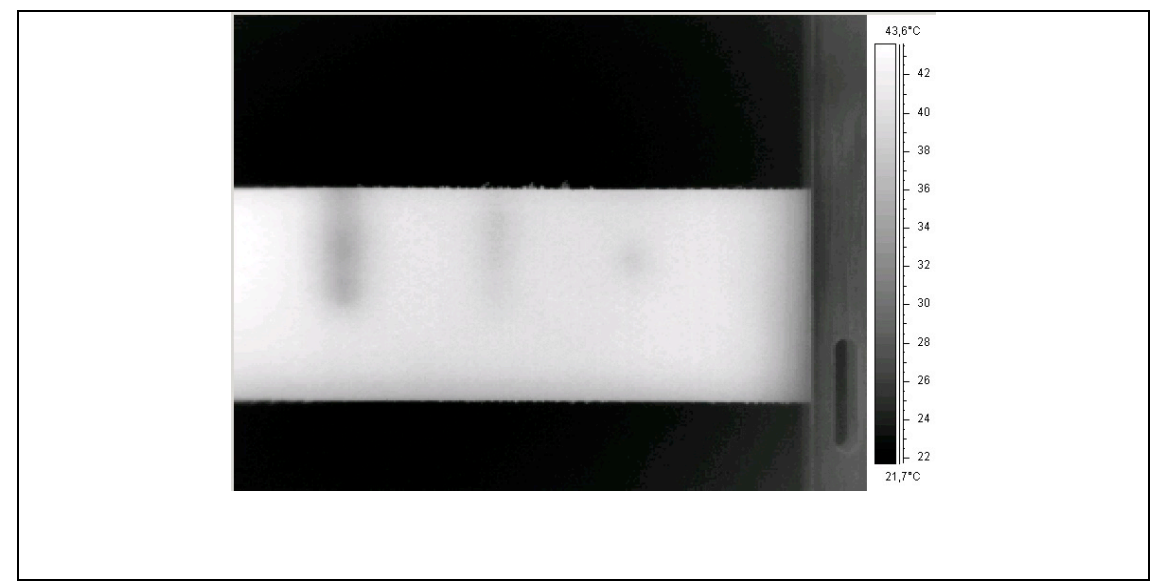

Figure 2 The structure of the composite used in the experimental phase and one of the corresponding thermographic images.

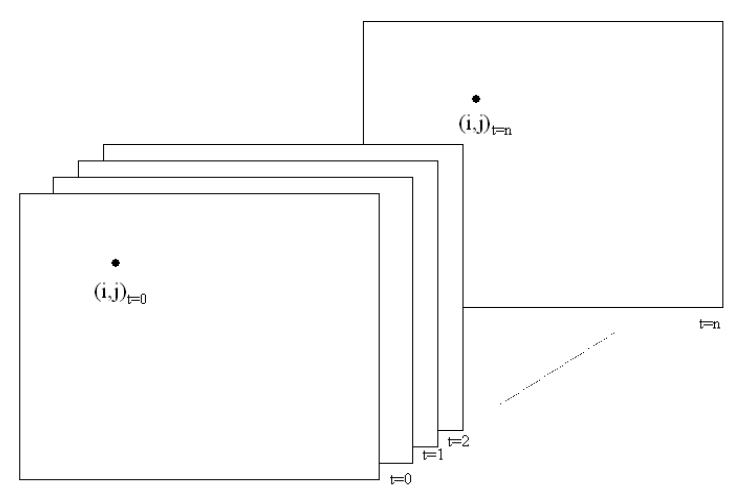

Figure 3- Extraction of the mono-dimensional signal from the temporal sequences of thermographic images 
http://dx.doi.org/10.21611/qirt.2006.016

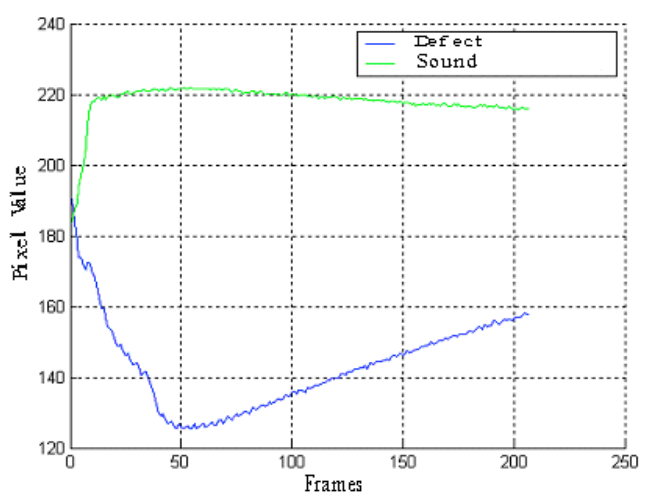

Figure 4 - Temperature variation of two points of the Nomex composite material

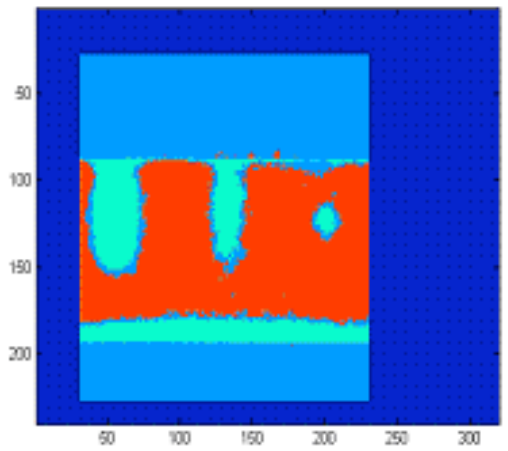

Figure 5- The classification results

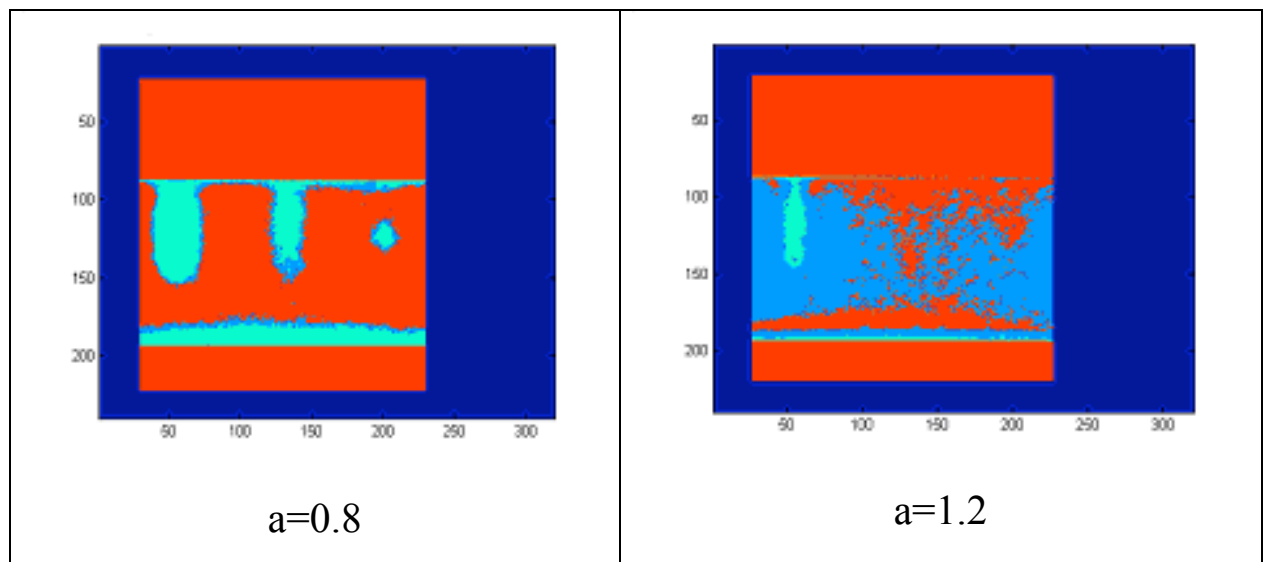

Figure 6- The classification results simulating linear lighting variations 